\title{
A Joint Subcarrier Assignment and Power Allocation with Statistical Delay QoS Provisioning in Full-Duplex OFDMA Systems
}

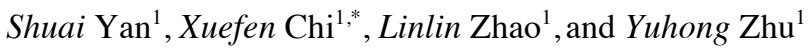 \\ ${ }^{1}$ Dept. of Communication Engineering, Jilin University, Nanhu Street 5372, Changchun, China
}

\begin{abstract}
In this paper, we explore a joint subcarrier assignment and power allocation with statistical delay QoS guarantees for full-duplex OFDMA systems. Effective capacity theory which evaluates wireless channel capacity from a novel view of link layer provides a theory basis for modeling the system capacity under the conditions of delay QoS constrains. After modeling the aggregate effective capacity of the system, we formulate joint subcarrier assignment and power allocation problem as a mixed integer programming problem, whose target is to maximize the aggregate effective capacity. To solve this integer programming problem, we allocate initial power on each subcarrier and decompose it into two subproblems, which are subcarrier allocation problem and power allocation problem respectively. Further, we propose an alternative algorithm to achieve joint subcarrier assignment and power allocation, which can meet the "subcarrier continuity" constraint of uplink resource allocation. Simulations have shown that the performance of our proposed scheme is favorite. Additionally, we investigate the effect of the residual selfinterference, inter-node interference (INI) and statistical delay QoS exponent on the performance of systems.
\end{abstract}

\section{Introduction}

Due to the shortage of radio spectrum, traditional radio communication systems suffer from limited channel capacity. Fortunately, full-duplex communication technologies provide an effective way to improve the spectrum efficiency via enabling a full-duplex node to transmit and receive simultaneously in the same frequency band [1-3].

The cellular communication system with full-duplex enable physical layer calls for a redesign of resource allocation algorithms in higher layers. The resource allocation algorithms in full-duplex OFDMA systems have been studied to maximize the sum-rate by assigning subcarriers and allocating transmission power under a limited power budget. The authors in [4-6] have formulated the joint problem of subcarrier assignment and power allocation in full-duplex OFDMA networks, and developed an iterative solution that achieves local Pareto optimality. The authors in [7] have proposed a three step subcarrier and power allocation algorithm to maximize the system throughput for the OFDMA full-

* Corresponding author: chixf@jlu.edu.cn 
duplex system. The authors in [8] focused on a full-duplex multi-user OFDMA cellular system, and they proposed a subcarrier allocation, power allocation and uplink and downlink user-pairing scheme to maximize the system sum-rate by integrating the dual method and the sequential parametric convex approximation method. For OFDMA systems, the uplink resource allocation is subject to subcarrier "continuity" constraints, which refers to the subcarriers or resource blocks (RBs) allocated to each user having to be continuous. However, the above-mentioned resource allocation algorithm for full-duplex OFDMA systems, no attempts have been devoted to propose resource allocation algorithm, which meet uplink resource allocation's "subcarrier continuity" constraint.

Furthermore, quality-of-service (QoS) guarantees play a critically important role in future communication networks. The next generation communication networks are required to provision high data rate and reliable service for delay sensitive applications. That is, more attention must be paid to evaluate the performance not only on the throughput, but also on the QoS guarantees.

Recently, the concept of statistic delay QoS in effective capacity theory has captured increasing attention of researchers. Different from the traditional Shannon capacity theory, effective capacity presents a novel perspective on the capacity of channel in terms of delay QoS metric in the link-layer [9-10], which can measure the throughput performance of the network under the statistic delay QoS constraint. However, for full-duplex OFDMA systems, how to maximize effective capacity while satisfying the given QoS requirement is an open problem.

In this paper, we consider a single-cell full-duplex OFDMA system, where the BS and nodes have power constraints and all nodes have QoS requirements. We aim to maximize the aggregate effective capacity by optimizing the uplink and downlink resource allocations while meeting the delay QoS requirements of transmission services in the presence of the full-duplex transmissions.

In order to obtain the aggregate effective capacity of the system, we define the subcarrier scheduling probability function to represent the subcarrier occupied by the node, and then get the probability of the service rate when the node occupies the subcarriers. Based on this, and combined with effective capacity theory, the effective capacity of a node under a subcarrier is modeled. Finally, we accumulate the effective capacity of all nodes to obtain the aggregate effective capacity of the system.

Relying on the derived system aggregate effective capacity, our joint subcarrier assignment and power allocation problem is formulated as a mixed integer programming problem with the target of maximizing the aggregate effective capacity. We target to support statistical delay QoS constraints. In view of the existing literature, the transmit power not only depends on the channel gain but also is influenced by statistical delay QoS. Therefore, we derive a QoS-driven power allocation strategy based on effective capacity theory. To solve this integer programming problem, we first allocate initial power on each subcarrier based on the given QoS-driven power allocation strategy, then the joint allocation problem can be decomposed into two sub-problems, which are subcarrier assignment problem and power allocation problem.

The subcarrier assignment is affected by the initial power, and also affects the final power allocation. Considering that subcarrier assignment and power allocation are intertwined, we solve these two sub-questions in two steps. First, for the uplink, we propose an iterative maximum expansion algorithm to solve the subcarrier allocation problem, which meets uplink resource allocation's "subcarrier continuity" constraints; for the downlink, we solve the subcarrier assignment problem based on a bipartite matching approach to make sure that a subcarrier is assigned only to one node. The uplink and downlink subcarrier assignment pattern is obtained, respectively. Second, according to the uplink and downlink subcarrier assignment pattern, we reallocate the power on each 
subcarrier based on the given QoS-driven power allocation strategy. In this way, an alternative algorithm to achieve joint subcarrier assignment and power allocation is formed.

The rest of this paper is organized as follows. The full-duplex OFDMA system model is described, and aggregate effective capacity maximization problem is formally formulated in Section 2. Our proposed subcarrier assignment and power allocation strategy are presented in Section 3. We evaluate our solution in comparison with other resource allocation schemes in Section 4. Finally, we conclude our paper in Section 5.

\section{System model and problem formulation}

In this paper, we consider a single-cell OFDMA system that consists of a full-duplex base station (BS), $N$ half-duplex uplink nodes and $M$ half-duplex downlink nodes. The system model is illustrated in Fig. 1. For the OFDMA system, the entire available bandwidth is equally divided into $S$ subcarriers, each of them with a bandwidth of $B . \mathbf{S}=\{1,2, \ldots, S\}$ is the sets of subcarriers, $\mathbf{N}=\{1,2, \ldots, N\}$ and $\mathbf{M}=\{1,2, \ldots, M\}$ are the sets of uplink nodes and downlink nodes, respectively. Each subcarrier is assumed to be perfectly orthogonal to any of other subcarriers, so there is no interference between different subcarriers. Owing to the BS operates in full-duplex mode, the BS can transmit and receive signals on the same frequency at the same time, there exists inter-node interference (INI) from uplink nodes to downlink nodes. Meanwhile, the BS is assumed to be imperfect fullduplex that exists residual self-interference. We regard the residual self-interference and INI as increasing the noise power[3], the INI of all nodes is the same, and let $\sigma_{\mathrm{BS}}$ and $\sigma_{\mathrm{INI}}$ be the residual self-interference of the BS and inter-node interference (INI), respectively.

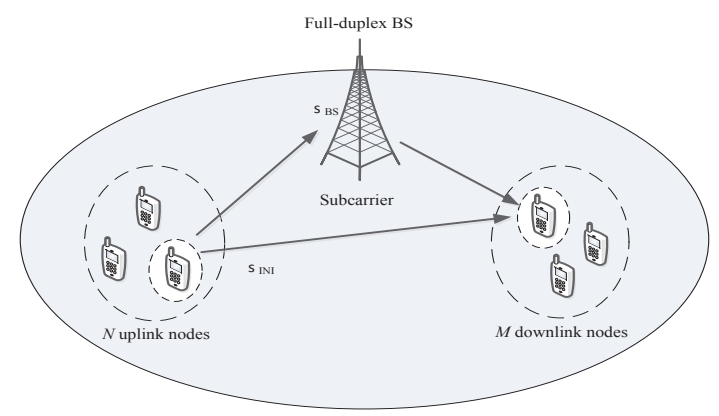

Fig. 1. A single-cell full-duplex OFDMA system

\subsection{Channel model}

In this scenario, we assume that the BS has the full knowledge of channel state information (CSI). We consider a block fading channel, and the channel fading gain includes the location-dependent path loss and the fast fading effect. The channel coefficient from node $n$ to BS, and that from BS to node $m$, over subcarrier $s$ are denoted by $h_{n, s}^{u}$ and $h_{m, s}^{d}$, respectively. Additionally, the transmit power from node $n$ to BS, and that from BS to node $m$, over subcarrier $s$ are denoted by $p_{n, s}^{u}$ and $p_{m, s}^{d}$, respectively. Then, the uplink and downlink transmit power vector are denoted by $\mathbf{P}^{u}:=\left\{p_{n, s}^{u}\right\}_{n \in \mathbf{N}, s \in \mathbf{S}}$ and $\mathbf{P}^{d}:=\left\{p_{m, s}^{d}\right\}_{m \in \mathbf{M}, s \in \mathbf{S}}$, respectively. Finally, we define $\mathbf{P}:=\left(\mathbf{P}^{u}, \mathbf{P}^{d}\right)$ as the power allocation vector. Owing to the residual self-interference and INI, the signal to interference plus noise power ratio (SINR) for uplink and downlink are $g_{n, s}^{u}=\left|h_{n, s}^{u}\right|^{2} /\left(\sigma_{\mathrm{BS}} N_{0}\right)$ and $g_{m, s}^{d}=\left|h_{m, s}^{d}\right|^{2} /\left(\sigma_{\mathrm{INI}} N_{0}\right)$, respectively, 
where $N_{0}$ denotes the receiver noise. Then, we define $\mathbf{G}:=\left\{g_{n, s}^{u}, g_{m, s}^{d}\right\}$ as the SINR vector. Thus, the rate of uplink transmission can be expressed as:

$$
R_{n, s}^{u}=B \cdot \log _{2}\left(1+P_{n, s}^{u} g_{n, s}^{u}\right)
$$

The rate of downlink transmission can be expressed as:

$$
R_{m, s}^{d}=B \cdot \log _{2}\left(1+P_{m, s}^{d} g_{m, s}^{d}\right)
$$

\subsection{Statistical delay-QoS guarantee}

Effective capacity theory, which is proposed by Wu and Negi in 2003 [11], is the dual problem of effective bandwidth. It characterizes the maximum constant arrival rate of a given service process under the statistical delay QoS constraint. Assume that $\{R[i], i=1$, $2, \ldots\}$ is an ergodic stochastic service process, $R[i]$ is the service rate of system in the $i$ slot. Then, the effective capacity of the service process is defined as:

$$
E C(\theta)=-\frac{1}{\theta} \log _{2}\left(\mathrm{E}\left\{e^{-\theta R[i]}\right\}\right)
$$

where the $\theta$ is the QoS exponent [12]. It should be noted that a small $\theta$ corresponds to a loose delay constraint, while a large $\theta$ indicates a stringent delay constraint.

According to (3), the uplink effective capacity can be expressed as:

$$
E C_{n, s}\left(\theta_{n}^{u}\right)=-\frac{1}{\theta_{n}^{u}} \log _{2}\left(e^{-\theta_{n}^{u}\left(R_{n, s}^{u}=0\right)}\left(1-\gamma_{n, s}\right)+e^{-\theta_{n}^{u}\left(R_{n, s}^{u}>0\right)} \gamma_{n, s}\right)
$$

the downlink effective capacity can be expressed as:

$$
E C_{m, s}\left(\theta_{m}^{d}\right)=-\frac{1}{\theta_{m}^{d}} \log _{2}\left(e^{-\theta_{m}^{d}\left(R_{m, s}^{d}=0\right)}\left(1-\gamma_{m, s}\right)+e^{-\theta_{m}^{d}\left(R_{m, s}^{d}>0\right)} \gamma_{m, s}\right)
$$

where $\theta_{n}^{u}$ and $\theta_{m}^{d}$ is the QoS exponent of uplink traffic and downlink traffic, respectively. $\gamma_{n, s}$ and $\gamma_{m, s}$ are the probability that node $n$ and node $m$ are scheduled on subcarrier $s$, respectively, if the node $n$ and node $m$ are scheduling, $R_{n, s}^{u}>0, R_{m, s}^{d}>0$; otherwise, $R_{n, s}^{u}=0, R_{m, s}^{d}=0$.

Then, the aggregate effective capacity of uplink and downlink can be expressed as:

$$
E C_{n, m, s}\left(\theta_{n}^{u}, \theta_{m}^{d}\right)=E C_{n, s}\left(\theta_{n}^{u}\right)+E C_{m, s}\left(\theta_{m}^{d}\right)
$$

For the OFDMA system, each subcarrier can only be assigned to a single node. To better express the node and subcarrier pairing, we define the uplink and downlink subcarrier assignment indication vector as $\mathbf{X}^{u}:=\left\{x_{n, s}^{u}\right\}_{n \in \mathbf{N}, s \in \mathbf{S}}$ and $\mathbf{X}^{d}:=\left\{x_{m, s}^{d}\right\}_{m \in \mathbf{M}, s \in \mathbf{S}}$, respectively, in which $x_{n, s}^{u}=1$ denotes that subcarrier $s$ is assigned to uplink node $n$ and $x_{m, s}^{d}=1$ denotes that subcarrier $s$ is assigned to downlink node $m$. Finally, we define $\mathbf{X}:=\left(\mathbf{X}^{u}, \mathbf{X}^{d}\right)$ as the subcarrier assignment vector. 


\subsection{Problem formulation}

In this paper, we let $P_{B S}$ and $P_{T}$ denote the total transmission power for the BS and for uplink node $n$, respectively. Thus, the joint subcarrier assignment and power allocation for aggregate effective capacity maximization is formulated as:

$$
\begin{aligned}
\text { P: maximize } & \sum_{n=1}^{N} \sum_{m=1}^{M} \sum_{s=1}^{S} x_{n, s}^{u} \cdot x_{m, s}^{d} \cdot E C_{n, m, s}\left(\theta_{n}^{u}, \theta_{m}^{d}\right) \\
\text { s.t. } \quad & \sum_{s=1}^{S} p_{n, s}^{u} \leq P_{T}, \forall n \in \mathbf{N} \\
& \sum_{m=1}^{M} \sum_{s=1}^{S} p_{m, s}^{d} \leq P_{B S}, \\
& P_{n, s}^{u}, P_{m, s}^{d} \geq 0, \forall n \in \mathbf{N}, m \in \mathbf{M}, s \in \mathbf{S} \\
& \sum_{n=1}^{N} x_{n, s}^{u} \leq 1, \sum_{m=1}^{M} x_{m, s}^{d} \leq 1, \forall s \in \mathbf{S} \\
& x_{n, s}^{u} \in\{0,1\}, \forall n \in \mathbf{N}, s \in \mathbf{S} \\
& x_{m, s}^{d} \in\{0,1\}, \forall m \in \mathbf{M}, s \in \mathbf{S}
\end{aligned}
$$

where the objective of the optimization Problem (7) is the sum of the effective capacity of in the system. Constraint (b) guarantees the limit of the total transmission powers for the node $n$. Constraint (c) guarantees the limit of the total transmission powers for the BS. Constraint (e), (f) and (g) guarantees each subcarrier is exclusively assigned to one uplink and downlink node.

\section{Subcarrier allocation and power allocation scheme}

The original problem $\mathrm{P}$ is an integer optimization problem. The subcarrier assignment and power allocation are intertwined: the subcarrier assignment is affected by the initial power, and also affects the final power allocation. In order to make the problem tractable, we first allocate initial power on each subcarrier based on the given QoS-driven power allocation strategy, then the joint allocation problem can be decomposed into two sub-problems, which are subcarrier assignment problem and power allocation problem. After that, an alternative algorithm that mixing the subcarrier assignment algorithm and QoS-driven power allocation strategy is proposed. We first describe QoS-driven power allocation strategy, and then consider the uplink and downlink subcarrier assignment algorithm.

\subsection{QoS-driven power allocation}

Given a certain determined subcarrier assignment pattern $\mathbf{X}^{*}$, the original optimization problem $\mathrm{P}$ is transformed into a power allocation problem. We target to support statistical delay QoS constraints, in view of the existing literature, the transmit power will depend on not only the channel gain but also the statistical delay QoS[10]. Then, the transmit power of node $n$ on subcarrier $s$ to BS can be derived as: 


$$
p_{n, s}^{u}=\left\{\begin{array}{cc}
\left.\beta_{n} \cdot\left(\frac{\alpha\left(\theta_{1}\right)^{\alpha\left(\theta_{2}\right)+1}}{\alpha\left(\theta_{2}\right)^{\alpha\left(\theta_{2}\right)} \cdot g_{n, s}^{u \alpha\left(\theta_{1}\right)+\alpha\left(\theta_{2}\right)}}\right)^{\frac{1}{\alpha\left(\theta_{1}\right)+\alpha\left(\theta_{2}\right)+1}}-\frac{1}{g_{n, s}^{u}}\right]^{+} \quad \text { if } x_{n, s}^{u}=1, \\
\text { if } x_{n, s}^{u}=0,
\end{array}\right.
$$

where $\alpha\left(\theta_{1}\right)=\theta_{n}^{u} / \ln 2$ is the constraint of statistical delay QoS for uplink, $\alpha\left(\theta_{2}\right)=\theta_{m}^{d} / \ln 2$ is the constraint of statistical delay QoS for downlink. The water level $\beta_{n}$ is a variable, which dependent on users' statistical delay QoS requirements and satisfying $\sum_{s=1}^{S} p_{n, s}^{u}=P_{T}$. Similarly, the transmit power of BS on subcarrier $s$ to node $m$ can be derived as:

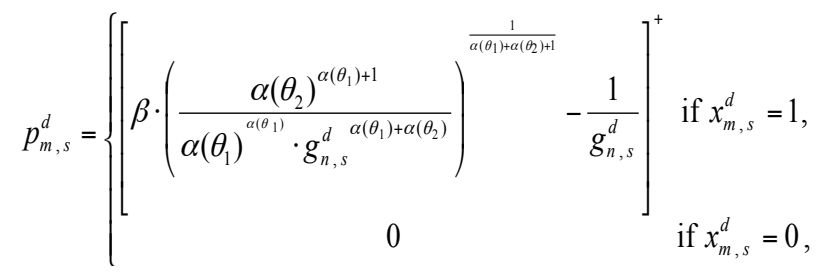

where $\beta$ can be numerically obtained by constraint $\sum_{m=1}^{M} \sum_{s=1}^{S} p_{m, s}^{d}=P_{B S}$.

\subsection{Subcarrier assignment}

Note that the constraints of $\mathrm{P}$ indicate the matching relationship between nodes and subcarriers, so we can transform $\mathrm{P}$ into a maximum weighted bipartite matching problem. We denoted the uplink and downlink weight matrix as $\mathbf{W}_{N \times S}$ and $\mathbf{W}_{M \times S}$, respectively. The elements of $\mathbf{W}_{N \times S}$ and $\mathrm{WM} \times \mathrm{S}$ are $w_{n, s}=E C_{n, s}\left(\theta_{n}^{u}\right)$ and $w_{m, s}=E C_{m, s}\left(\theta_{m}^{d}\right)$, respectively.

\subsubsection{Uplink subcarrier assignment}

In this paper, we propose an iterative maximum expansion (IME) algorithm to solve the uplink subcarrier allocation problem, which meet uplink resource allocation's "subcarrier continuity" constraint. The basic idea of IME is that the maximum value is selected from all the weight values of each user on each subcarrier, and the subcarrier corresponding to the maximum weight value is assigned to the corresponding user, and then the subcarrier position is centered and to expand the resources for this user on both sides, until the extended weight of this user on the subcarrier is less than the other user and get the uplink maximum weighted matching $F^{u}$.

\subsubsection{Downlink subcarrier assignment}

The Hungarian algorithm is an algorithm for solving assignment problems, the basic idea is to transform the original efficiency matrix $\mathrm{C}$ into a new efficiency matrix $\overline{\mathrm{C}}$, so that the new matrix $\overline{\mathrm{C}}$ contains many zero elements, but the optimal solution does not change. In the new efficiency matrix $\overline{\mathrm{C}}$, If you can find $\mathrm{n}$ (equal to the order of the matrix) zero elements which different rows and different columns, you can make their corresponding $x_{i j}$ is equal to 1 , the other $x_{i j}$ is equal to 0 , then the solution must be the program Optimal solution. 
However, the Hungarian algorithm requires that the number of elements on both sides be equal, and in fact, the number of subcarriers is usually greater than the number of nodes. To this end, we re-use the Hungarian algorithm until all of the subcarriers are assigned to the nodes and get the downlink maximum weighted matching $F^{d}$.

The detailed algorithm can be summarized as the following procedures. In the first step, calculate $p_{n, s}^{u}$ and $p_{m, s}^{d}$ according to (8) and (9), respectively. Then, calculate $E C_{n, s}\left(\theta_{n}^{u}\right)$ and $E C_{m, s}\left(\theta_{m}^{d}\right)$ according to (4) and (5) based on the results of $p_{n, s}^{u}$ and $p_{m, s}^{d}$, respectively. In the second step, adopt the IME algorithm to get the uplink maximum weighted matching $F^{u}$ with the weight matrix $\mathbf{W}_{N \times S}$; adopt the Hungarian algorithm to get the downlink maximum weighted matching $F^{d}$ with the weight matrix $\mathbf{W}_{M \times S}$. Then, we can get the subcarrier assignment $\mathbf{X}^{*}:=\left(\mathbf{X}^{u}, \mathbf{X}^{d}\right)$, where $\mathbf{X}^{u}=F^{u}$ and $\mathbf{X}^{d}=F^{d}$. Finally, allocate uplink power $\widetilde{p}_{n, s}^{u}$ by (8) and downlink power $\widetilde{p}_{m, s}^{d}$ by (9) according to $\mathbf{X}^{*}$, so, we can get the power allocation $\widetilde{\mathbf{P}}$.

\section{Simulation results}

In this section, we conduct simulation experiments to evaluate the performance of the proposed resource allocation scheme for full-duplex OFDMA systems under the LTE specifications[5]. We assume that the number of uplink and downlink nodes is equal to $N$. The detailed simulation parameters are listed in Table 1 . In addition to the path loss $P_{\text {loss }}$ $(\mathrm{dB})$, it is obtained from the modified Hata urban propagation model, which is calculated as:

$$
P_{\text {loss }}(d)=69.55+26.16 \cdot \log f-13.83 \cdot \log h_{B S}-C_{H}(f)+\left(44.9-6.55 \cdot \log h_{B S}\right) \log d
$$

where $d(\mathrm{~km})$ is the distance between BS and nodes, $h_{B S}(\mathrm{~m})$ is the height of the BS, $f(\mathrm{MHz})$ is the center frequency. $C_{H}(f)$ is the antenna height correlation factor, which is defined as:

$$
C_{H}(f)=0.8+(1.1 \cdot \log f-0.7) h-1.56 \cdot \log f
$$

where $h(\mathrm{~m})$ is the height of the node. We assume that each node is placed at an equal distance $d$ from the BS.

Table 1. Simulation parameters.

\begin{tabular}{|l|l|}
\hline Parameter & Settings \\
\hline Carrier center frequency & $f=2.1 \mathrm{GHz}$ \\
\hline Subcarrier bandwidth & $\mathrm{B}=180 \mathrm{kHz}$ \\
\hline Noise power & $N_{0}=-119 \mathrm{dBm}$ \\
\hline Maximum BS power & $P_{B S}=43 \mathrm{dBm}$ \\
\hline Maximum Node power & $P_{T}=24 \mathrm{dBm}$ \\
\hline The height of the BS & $h_{B S}=30 \mathrm{~m}$ \\
\hline The height of the node & $h=1.5 \mathrm{~m}$ \\
\hline
\end{tabular}

Fig. 2 illustrates the effect of the number $N$ of nodes on the aggregate effective capacity when $S=30, d=200 \mathrm{~m}, \sigma_{\mathrm{BS}}=1 \mathrm{~dB}$ and $\sigma_{\mathrm{INI}}=3 \mathrm{~dB}$. For any $\mathrm{n} \in \mathrm{N}$ and $\mathrm{m} \in \mathrm{M}$, we let $\theta_{n}^{u}=\theta_{m}^{d}=10^{-5}$. Observed from Fig. 2, with more nodes, the aggregate effective capacity of each scheme increases towing to the multi-user diversity. Our resource allocation scheme can achieve a greater aggregate effective capacity than the iterative allocation scheme[6] does, and the advantages of our resource allocation scheme would be greater with the increase of the number of nodes. 
Fig. 3 demonstrates the impact of statistical delay QoS requirements on the aggregate effective capacity when $S=30, N=30$ and $d=200 \mathrm{~m}$. Observed from Fig. 3, the aggregate effective capacity reduces upon increasing the downlink required statistical delay QoS exponent, the full-duplex OFDMA system is sensitive to the statistical delay QoS requirements, and the effective capacity degrades rapidly for $\theta_{m}^{d}$ in the range $10^{-4}$ to $10^{-2}$.

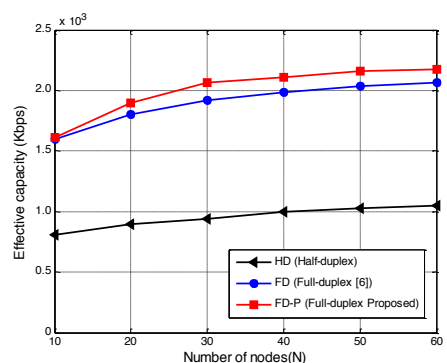

Fig. 2. Performance comparison according to $\mathrm{N}$ values

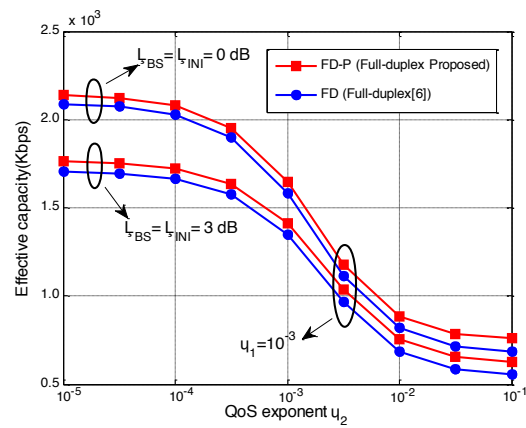

Fig. 4. Performance comparison according to downlink statistical delay QoS values With different interference

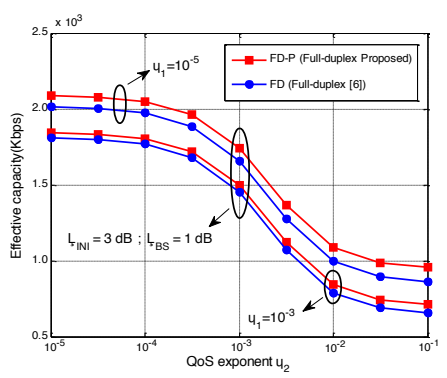

Fig. 3. Performance comparison of different uplink statistical delay QoS requirements

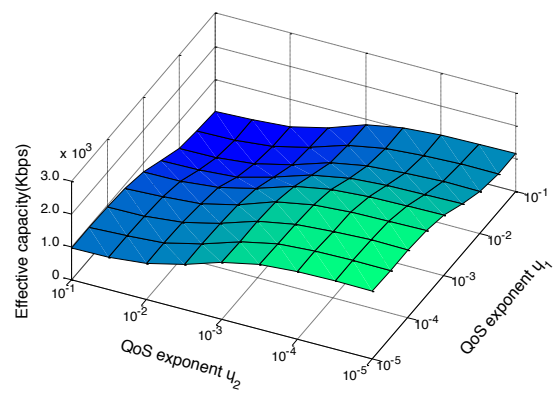

Fig. 5. The impact of heterogeneous statistical delay QoS requirements on the aggregate effective capacity

Fig. 4 demonstrates the impact of the statistical delay QoS requirements and interference on the aggregate effective capacity when the uplink required QoS exponent $\left(\theta_{n}^{u}\right)$ is $10^{-3}, S=30, N=30$ and $d=200 \mathrm{~m}$. Observed from Fig. 4, for any statistical delay QoS requirements, the aggregate effective capacity reduces upon increasing the residual self-interference or INI. When statistical delay QoS exponent is very small, residual selfinterference or INI has a large effect on system aggregate effective capacity, this is because with the statistical delay QoS exponent gradually smaller, the statistical delay QoS requirements is very loose, system aggregate effective capacity is largely unconstrained by statistical delay.

Fig. 5 evaluates the aggregate effective capacity versus uplink required QoS exponent $\left(\theta_{n}^{u}\right)$ and downlink required QoS exponent $\left(\theta_{m}^{d}\right)$ corresponding to heterogeneous statistical QoS provisioning when $\sigma_{\mathrm{BS}}=1 \mathrm{~dB}$ and $\sigma_{\mathrm{INI}}=3 \mathrm{~dB}$. As shown in Fig. 5, the aggregate effective capacity decreases with the uplink required QoS exponent and the downlink required QoS exponent. In addition, when statistical delay QoS is very loose, the system can get the maximum aggregate effective capacity. 


\section{Conclusion}

In this paper, we investigated the joint subcarrier assignment and power allocation problem with statistical delay QoS guarantees for users in full-duplex OFDMA systems. Our joint subcarrier assignment and power allocation problem was formulated as a mixed integer programming problem whose target is to maximize the aggregate effective capacity. In order to make this integer programming problem tractable, we allocate initial power on each subcarrier and decompose it into two sub-problems, which are subcarrier allocation problem and power allocation problem respectively. Based on IME algorithm, Hungarian algorithm and QoS-driven power allocation strategy, we proposed a new resource allocation scheme to solve this aggregate effective capacity maximization problem with polynomial computational complexity. The numerical simulations demonstrated that our proposed scheme was more effective to the system effective capacity than the iterative allocation scheme in [6], especially when the number of subcarriers and nodes was large. In addition, when statistical delay QoS was very loose, the system can get the maximum aggregate effective capacity, and compared with the very stringent requirements for statistical delay QoS, residual self-interference or INI has a greater impact on system aggregate effective capacity.

\section{References}

1. J.I. Choi, M. Jain, K. Srinivasan, P. Levis, S. Katti, MOBICOM, pp. 1-12, (2010)

2. M. Jain, J.I. Choi, T. Kim, MOBICOM, pp. 301-312, (2011)

3. D. Bharadia, E. Mcmilin, S. Katti, CCR, 43, 4, pp. 375-386, (2013)

4. C. Nam, C. Joo, S. Bahk, ICTC IEEE, pp. 924-927, (2014)

5. C. Nam, C. Joo, S. Bahk, IEEE Transactions on Wireless Communications, 14, 6, pp. 3018-3119, (2015)

6. C. Nam, C. Joo, S. Bahk, IEEE ICC, pp. 3885-3890, (2015)

7. A.C. Cirik, K. Rikkinen, R. Yue, T. Ratnarajah, EuCNC, pp. 11-15, (2015)

8. Y. Jiang, H. Chen, F.C.M. Lau, P. Wang, Y. Li, Transactions on Emerging Telecommunications Technologies, pp. 1-19, (2015)

9. W. Cheng, X. Zhang, H. Zhang, IEEE ICC, pp. 5286-5290, (2012)

10. W. Cheng, X. Zhang, H. Zhang, Computer Communications. IEEE, pp. 55-63, (2015)

11. D. Wu, R. Negi, IEEE Transactions on Wireless Communications, 2, 4, pp. 630-643, (2003)

12. J. Tang, X. Zhang, IEEE Transactions on Wireless Communications, 6, 8, pp. 30583068, (2007) 